
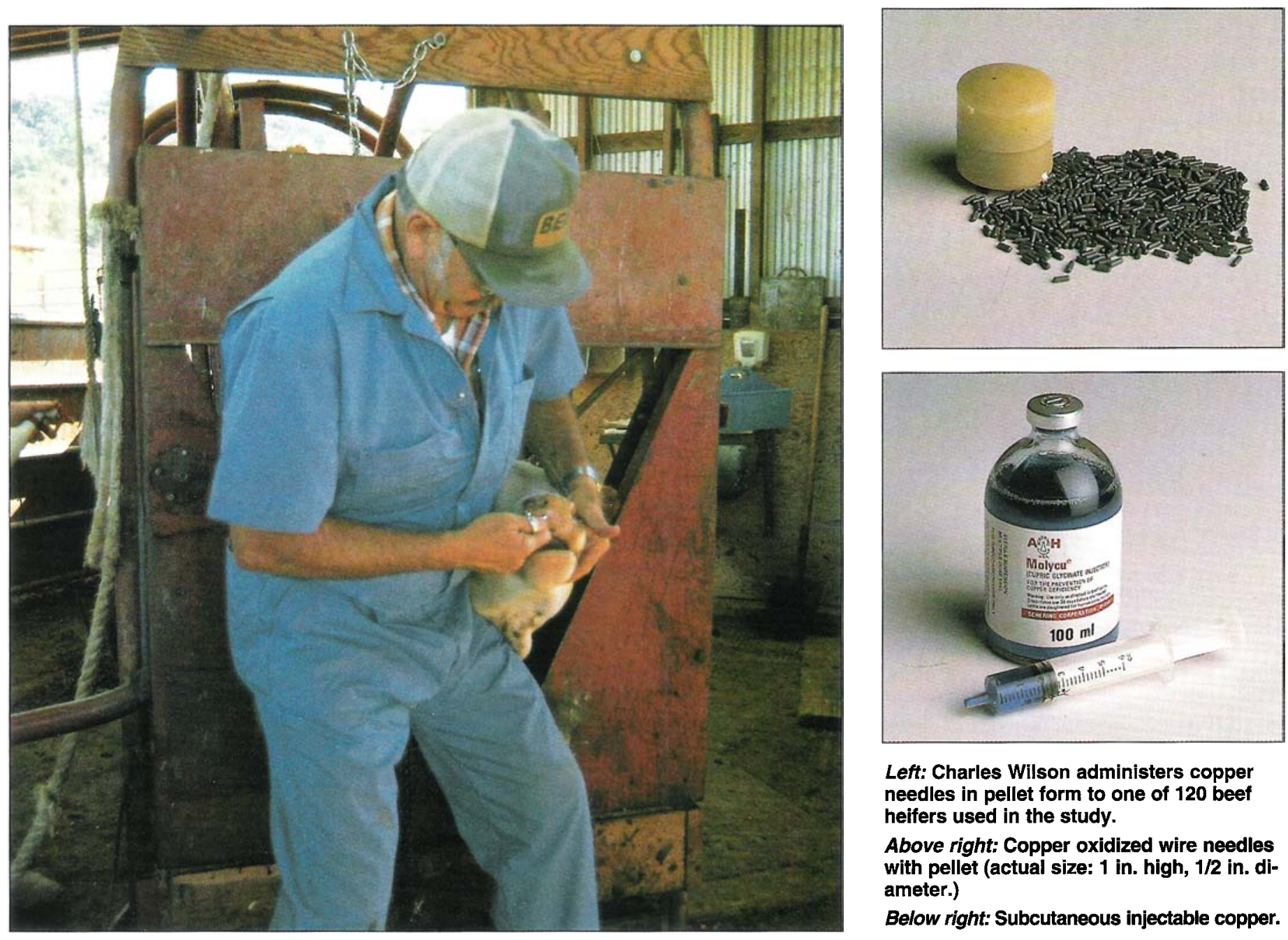

Left: Charles Wilson administers copper needles in pellet form to one of 120 beef heifers used in the study.

Above right: Copper oxidized wire needles with pellet (actual size: 1 in. high, 1/2 in. diameter.)

Below right: Subcutaneous injectable copper.

\title{
Cupric-oxide needles effective as oral copper supplement in cattle
}

\author{
John R. Dunbar $\quad$ James G. Morris $\quad \square \quad$ Ben B. Norman $\quad \square \quad$ A. J. Jenkins \\ Charles B. Wilson $\square$ John M. Connor
}

Increasing copper levels in 120 growing beef heifers, ages 6 to 9 months, was attempted with copper injections and with oral administration of cupric-oxide needles. The weaned Hereford heifers were randomly allocated to three groups, including one with no supplementation. Study results indicate that oral administration of copper-wire particles was effective, cheap, safe and convenient in preventing or treating copper deficiency. Such a deficiency can retard growth in cattle.
In grazing areas of endemic copper deficiency in California, young cattle have responded to supplements of copper in their diets by increasing their feed intake and growth rate, and efficiently improving their feed conversion.

The biological need for copper in animal diets was first shown with anemic, milk-fed rats in 1928. Since then, the role of copper in supporting animal growth, normal iron metabolism and fertility, and in preventing bovine falling disease, hair depigmentation and fragile bones, has been demonstrated. Customarily, copper is administered in copper-containing mineral mixes, mineral blocks or liquid supplements and by injection.

The feasibility of treating animals orally with doses of cupric-oxide wire needles developed in Australia, where the needles, enveloped in capsules made of a degradable blend of polymers, were administered with balling guns. A steady increase in desirable levels of liver copper resulted. (Too much copper can be toxic.) The needles did not pass through the digestive tract; they lodged primarily between the abomasal folds and released copper under conditions favorable for absorption.

Our trial was designed to determine whether supplementation with copper-oxide needles would increase liver copper levels with no deleterious effects on experimental animals.

\section{Methods}

The University of California's Sierra Foothill Range Field Station was the site of 


\section{Sierra Copper Trial 1986}

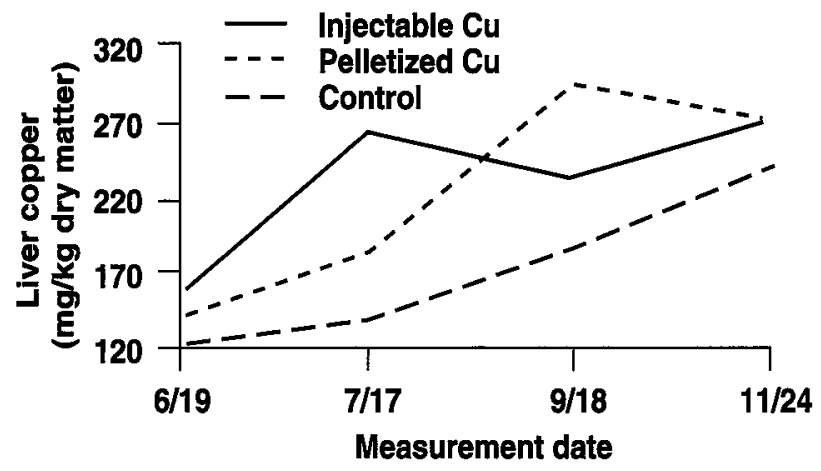

increased $(\mathrm{P}<.05)$ liver copper levels, compared with the other two groups. Oxidized copper-wire particles produced significantly $(\mathrm{P}<.05)$ higher levels of liver copper at 28 days, compared with controls. At 91 days, there was also evidence that the copper-wire particles produced significantly $(\mathrm{P}<.05)$ higher liver copper levels,

Fig. 1. Levels of liver copper obtained using injectable and pellet forms of copper amendments.

the experiment. One hundred and twenty Hereford heifers, ages 6 to 9 months and averaging $210 \mathrm{~kg}$ (463 pounds), were randomly assigned to three treatment groups. Forty heifers were left untreated, 40 were given $10 \mathrm{~g}$ of orally administered, oxidized copper-wire particles in a soluble capsule, and the remaining 40 received a subcutaneous injection of Molycu (120 mg of copper as cupric glycinate). Liver biopsies and blood samples were collected from a randomized sample of 13 heifers in each treatment group at day 0 and at 28,91 and 158 days. Blood and liver samples were analyzed for copper, selenium and iron.

\section{Analysis}

Data were analyzed with NCSS statistical software using analysis of variance (ANOVA) procedures for independent responses and Duncan's multiple comparison test.

Results are given in tables 1 and 2. At 28 days, the copper injection significantly jected heifers. Although liver concentrations in control heifers were generally below those in treated heifers, they were indicative of adequate copper status (we started with heifers that had no copper deficiency problems). At 158 days, no significant difference in liver copper status existed among the groups. Mean plasma copper values were not altered by treatment during the study. Selenium and copper concentrations in blood and liver were not altered by treatment. The copper injection caused severe tissue reactions at the site of injection (brisket) in 30 out of 40 heifers. Blood selenium and iron values were not altered by copper treatment.

Liver concentration of copper in cattle is sensitive to the quantity of supplement given; the chemical form of the copper; the available copper, molybdenum, selenium and iron content of the diet; the initial copper status of the animal, and the time of treatment. From this study it appears that the period of protection from copper deficiency offered by copper-oxide wire is at least 91 days. The period of protection with copper-deficient cattle may be longer. The major disadvantage of using injectable compared with controls and copper-in-

\begin{tabular}{|c|c|c|c|c|c|}
\hline \multicolumn{6}{|c|}{ TABLE 1. Liver copper levels over time } \\
\hline \multirow[b]{2}{*}{ Treatment } & \multirow{2}{*}{ Heifers } & \multicolumn{4}{|c|}{ Days after treatment } \\
\hline & & 0 & 28 & 91 & 158 \\
\hline & no. & ............ & .....mg/ & atter...... & ........... \\
\hline Control & 13 & $123 a$ & $138 \mathrm{a}$ & $184 a$ & $233 a$ \\
\hline Copper injection & 13 & $152 a$ & $258 b$ & $228 a$ & $263 a$ \\
\hline Copper pellet & 13 & $142 \mathrm{a}$ & $194 \mathrm{C}$ & $296 b$ & $264 a$ \\
\hline \multirow{2}{*}{\multicolumn{6}{|c|}{$\begin{array}{l}\text { Note: Means in the same column with the same letters are not significantly different at the }(P<0.05) \text {, leve } \\
\text { signficance. }\end{array}$}} \\
\hline & & & & & \\
\hline & \multirow[b]{2}{*}{ Heifers } & \multicolumn{4}{|c|}{ Days after treatment } \\
\hline & & 0 & 28 & 91 & 158 \\
\hline & no. & \multicolumn{4}{|c|}{. } \\
\hline Control & 39 & 1.03 & 0.89 & 0.93 & 0.98 \\
\hline Copper injection & 39 & 1.02 & 0.94 & 0.89 & 0.94 \\
\hline Copper pellet & 39 & 1.02 & 1.01 & 0.96 & 0.99 \\
\hline
\end{tabular}

